\title{
CRECIMIENTO DE CULTIVARES DE GUAYABO EN CONDICIONES DE ESTRÉS POR SALINIDAD
}

\section{GROWTH OF GUAVA TREE CULTIVARS UNDER SALINITY STRESS CONDITIONS}

\author{
Francisco J. Inda-Romero', Beatriz G. Arrieta-Ramos ${ }^{2}{ }^{*}$ \\ Álvaro Can-Chulím², Rubén Bugarín-Montoya ${ }^{2}$ y Gelacio Alejo-Santiago ${ }^{2}$
}

\begin{abstract}
'Universidad Autónoma de Nayarit, Unidad Académica de Agricultura, Programa en Ciencias Biológico Agropecuarias, Xalisco, Nayarit, México. ²Universidad Autónoma de Nayarit, Unidad Académica de Agricultura, Xalisco, Nayarit, México.
\end{abstract}

*Autor de correspondencia (g-arrieta@hotmail.com)

\section{RESUMEN}

El guayabo (Psidum guajava L.) es un cultivo rústico que puede ser utilizado en zonas con suelos marginales. Esta investigación se planteó con el objetivo de evaluar la respuesta del guayabo a estrés salino durante la etapa de crecimiento. Las plantas se obtuvieron de estacas enraizadas de los cultivares Calvillo Siglo XXI y Merita. El experimento se realizó bajo un sistema hidropónico a raíz flotante, en solución Steiner 50 \%. Se evaluaron cinco condiciones de salinidad y se ajustó la conductividad eléctrica (CE) con $\mathrm{NaCl}$ a 2.0, 2.5, 3.0 y $3.5 \mathrm{dS} \mathrm{m}^{-1}$, y el testigo a $1.2 \mathrm{dS} \mathrm{m}^{-1} \sin \mathrm{NaCl}$. El diseño experimental fue completamente al azar con cinco repeticiones. Las variables evaluadas fueron número y longitud de brotes, número de hojas por brote, vida media de hoja, volumen de agua transpirada, contenido de clorofila, área foliar, longitud de raíces, materia fresca y seca en hojas, tallo y raíz, concentración foliar y radical de $\mathrm{P}, \mathrm{Ca}, \mathrm{K}$ y Na, y densidad y diámetro longitudinal de estomas. El cultivar Calvillo Siglo XXI superó a Merita en contenido de clorofila (36.35 vs 32.74 unidades SPAD); Merita, en cambio, presentó mayor concentración de $\mathrm{P}(0.1306$ vs $0.1106 \%)$ y Ca (1.0514 vs $0.7463 \%)$ en hoja. La salinidad disminuyó la vida media de la hoja de 74 a 42 d; las hojas presentaron en promedio mayor concentración de K que la raíz (2.372 vs $0.966 \%$ ). A 3.5 dS $\mathrm{m}^{-1}$ de CE la concentración de $K$ disminuyó en $31.8 \%$ en hojas y $55.5 \%$ en raíz, y la concentración de Na en hojas incrementó en $68.6 \%$ en comparación con el testigo. La capacidad de los dos cultivares de mantener alta concentración de $\mathrm{K}$ en hojas, así como el transporte del Na hacia las hojas, incrementó la resistencia a la salinidad de los cultivares en los niveles evaluados. La salinidad no tuvo efecto significativo en el crecimiento de las plantas de guayabo.

Palabras clave: Psidum guajava, conductividad eléctrica, estomas, vida media de la hoja.

\section{SUMMARY}

Guava tree (Psidum guajava L.) is a rustic fruit crop that can be used in areas with marginal soils. This research evaluated the response of guava tree to saline stress during the growth stage. The plants were obtained from rooted cuttings of the Calvillo Siglo XXI and Merita cultivars. The experiment was conducted under a floating root hydroponic system, in $50 \%$ Steiner solution. Five salinity conditions were evaluated, and the electrical conductivity was adjusted with $\mathrm{NaCl}$ to $2.0,2.5,3.0$ and $3.5 \mathrm{dS} \mathrm{m}^{-1}$, and the control to $1.2 \mathrm{dS}$ $\mathrm{m}-1$ without $\mathrm{NaCl}$. The experimental design was completely randomized with five replications. The evaluated variables were number and length of sprouts, number of leaves per sprout, average leaf lifespan, transpired water volume, chlorophyll content, leaf area, root length, fresh and dry matter in leaves, stem and root. Additionally, leaf and root concentration of $\mathrm{P}, \mathrm{Ca}, \mathrm{K}$ and $\mathrm{Na}$ and density and longitudinal diameter of stomata were determined. Calvillo Siglo XXI cultivar outperformed Merita in chlorophyll content (36.35 vs 32.74 SPAD units); in contrast, Merita presented higher concentration of $P$ $(0.1306$ vs $0.1106 \%)$ and $\mathrm{Ca}(1.0514$ vs $0.7463 \%)$ in leaf. Salinity decreased the average leaf lifespan from 74 to $42 \mathrm{~d}$. Leaves contained, on average, a higher $\mathrm{K}$ concentration than roots (2.372 vs $0.966 \%$ ). At $3.5 \mathrm{dS} \mathrm{m}^{-1} \mathrm{EC}$, the $\mathrm{K}$ concentration decreased by $31.8 \%$ in leaves and $55.5 \%$ in roots, and the $\mathrm{Na}$ concentration in leaves increased by $68.6 \%$ compared to the control. The capacity of the two cultivars to maintain high $\mathrm{K}$ concentration in leaves, as well as the transport of $\mathrm{Na}$ to leaves, increased their salinity resistance at the evaluated levels. Salinity had no significant effect on the growth of guava plants.

Index words: Psidum guajava, average leaf lifespan, electrical conductivity, stomata.

\section{INTRODUCCIÓN}

El guayabo (Psidium guajava L.) es una especie importante en México, ya que de éste se pueden aprovechar el fruto, las flores, hojas y raíces; además, es un cultivo muy rústico, resiste la sequía, tolera déficits nutrimentales y puede sobrevivir con pocos cuidados en condiciones desfavorables, por lo que puede explotarse en tierras marginales. Zacatecas y Aguascalientes, que durante muchos años fueron los primeros productores a nivel nacional, no cuentan con las condiciones edafoclimáticas adecuadas para su producción (SIAP, 2018).

La salinidad del suelo es un problema para la agricultura, ya que limita la producción. Actualmente, alrededor del 6 $\%$ de la superficie terrestre en el mundo está afectada por sales y este número aumenta debido a regímenes hídricos inadecuados o uso de agua salina en el riego (Munns y Tester, 2008). En México, el problema de salinidad se presenta en las zonas áridas y semiáridas, y a lo largo de las costas.

En la mayoría de suelos y aguas salinas el catión soluble 
que predomina es el $\mathrm{Na}^{+}$. Debido a que la acumulación de $\mathrm{Na}$ intercelular es negativa en el metabolismo celular, la mayoría de cultivos exhiben hipersensibilidad a los ambientes salinos (Cha-Um y Kirdmanee, 2009). Las sales en la rizosfera pueden provocar desbalance nutrimental, tanto por una disminución en la absorción de nutrimentos como por desbalance iónico, debido a la acumulación de $\mathrm{Na}^{+}$y $\mathrm{Cl}^{-}$en las células, lo que reduce la absorción de $\mathrm{K}^{+}$ $\mathrm{Ca}^{2+}, \mathrm{Mn}^{+}, \mathrm{NO}_{3}{ }^{-}$y $\mathrm{PO}_{4}{ }^{3-}$ (Nawaz et al., 2010). En condiciones salinas el $\mathrm{Na}^{+}$compite con el $\mathrm{K}^{+}$en los cotransportadores $\mathrm{Na}^{+}-\mathrm{K}^{+}$, y puede bloquear transportadores específicos de $\mathrm{K}^{+}$de las células de las raíces (Zhu, 2003). El $\mathrm{K}^{+}$es el ion más importante en la planta, ya que está implicado en el balance hídrico y en el potencial osmótico en la estela de la raíz, lo que provoca alteraciones en la presión de turgencia y el transporte de solutos en el xilema (Marschner, 2002).

Las plantas pueden responder al estrés salino con mecanismos fisiológicos a nivel molecular, celular y de organismo, que incluyen cambios en el desarrollo y morfología, ajuste en el transporte iónico en relación con la concentración, expulsión y secuestro de iones, regulación de los sistemas de defensa antioxidantes, cambios en la expresión de genes y proteínas, y otros mecanismos metabólicos (Fuentes et al., 2007; Com. Pers.)'. El ajuste osmótico es un mecanismo que permite a la planta mantener la absorción de agua y la presión de turgencia en condiciones de estrés hídrico; se basa en la acumulación activa de solutos que pueden ser iones como $\mathrm{Na}^{+} \mathrm{y} \mathrm{K}^{+}, \mathrm{y}$ en la síntesis de compuestos orgánicos, entre los que se encuentran prolina, colina, glicina-betaína, betaína, polioles y azúcares solubles (Zhu, 2003).

La obtención de variedades tolerantes a salinidad, o portainjertos tolerantes a estas condiciones, puede ser una solución al problema, ya que debido a la variabilidad genética, la salinidad afecta de forma diferenciada a variedades de la misma especie (Ruiz et al., 2014).

En el caso del guayabo, existen algunos reportes sobre la tolerancia a la salinidad. Sá et al. (2016) probaron el efecto de cinco niveles de salinidad en el agua de riego $(0.6,1.2,1.8$, 2.4 y $3.0 \mathrm{dS} \mathrm{m}^{-1}$ ) por $30 \mathrm{~d}$ sobre la germinación de semillas de tres portainjertos de guayabo (Criolla, Paluma y Ogawa) y encontraron que el aumento en la concentración de sales restringe la germinación de las semillas, el crecimiento y la acumulación de biomasa y el mayor efecto ocurrió a niveles superiores a $1.8 \mathrm{dS} \mathrm{m}^{-1}$. El número de hojas y biomasa seca total fueron las variables más afectadas por el estrés por sales.

'Fuentes A. L., M. Sosa C. y Y. Pérez H. (2007) Aspectos fisiológicos y bioquímicos del estrés salino en plantas. Monografía. Facultad de Agronomía, Universidad de Matanzas "Camilo Cienfuegos" Matanzas, Cuba. $17 \mathrm{p}$.
Casierra-Posada (2006) evaluó plántulas de guayabo cv. Palmira ICA-1 de $90 \mathrm{~d}$ con seis hojas en condiciones salinas inducidas con $\mathrm{NaCl}\left(1.9,3.8,6.2,8.6\right.$ y $\left.11.5 \mathrm{dS} \mathrm{m}^{-1}\right)$ y observó que los tratamientos redujeron el área foliar hasta en $61.8 \%$; sin embargo, éstos indujeron pocos cambios en el contenido de materia seca, lo que sugiere que este cultivar es relativamente tolerante a la salinidad por $\mathrm{NaCl}$.

De la misma forma, en plantas de guayabo cv. Allahabad Safeda de un año de edad se encontró un umbral de tolerancia a la salinidad de aproximadamente $1.5 \mathrm{dS}$ $\mathrm{m}^{-1}$, lo que es idóneo para el cultivo comercial en suelos moderadamente salinos. La tolerancia de este cultivar se atribuyó a la absorción preferente de $\mathrm{K}^{+}$por la raíz (Singh et al., 2016). En este contexto, el objetivo del presente estudio consistió en evaluar la respuesta de dos cultivares de guayabo a estrés salino en etapa de crecimiento bajo invernadero en un sistema de cultivo hidropónico en solución nutritiva.

\section{MATERIALES Y MÉTODOS}

\section{Sitio experimental}

La presente investigación se realizó en la Unidad Académica de Agricultura de la Universidad Autónoma de Nayarit, dentro de un invernadero tipo cenital con cobertura de polietileno blanco lechoso y malla antiáfidos en las ventanas laterales, con ubicación geográfica a $21^{\circ}$ $25^{\prime} 40^{\prime \prime} \mathrm{N}, 104^{\circ} 53^{\prime} 30^{\prime \prime}$ O y $984 \mathrm{~m}$ de altitud. La temperatura máxima promedio fue de $41{ }^{\circ} \mathrm{C}$, mínima de $18{ }^{\circ} \mathrm{C}$ y humedad relativa promedio de $60 \%$ durante los meses de marzo a junio.

\section{Material vegetal}

Se evaluaron los cultivares Calvillo Siglo XXI y Merita, a partir de estacas de $30 \mathrm{~cm}$ de largo y al menos $0.5 \mathrm{~cm}$ de diámetro, obtenidas de un huerto experimental establecido en el Instituto Nacional de Investigaciones Forestales, Agrícolas y Pecuarias (INIFAP) en Santiago Ixcuintla, Nayarit y enraizadas en una cama con agrolita. Cuando las estacas presentaron callo y comenzaron a desarrollar raíces se regaron con solución nutritiva universal de Steiner al 50 \% (Steiner, 1984) dos veces por semana; posteriormente, las plantas obtenidas se establecieron en un sistema hidropónico con raíz flotante, en contenedores con capacidad de $12 \mathrm{~L}$, que fueron llenados con $10 \mathrm{~L}$ de solución nutritiva universal de Steiner. El sistema de aireación se instaló con bombas para pecera de $0.175 \mathrm{~kg}$ $\mathrm{cm}^{-2}$, que se mantuvieron encendidas las $24 \mathrm{~h}$ del día. Los tratamientos salinos correspondientes se aplicaron dos semanas después del trasplante. La solución se renovaba cuando se consumía el $25 \%$. 


\section{Tratamientos, diseño y unidad experimental}

Se utilizó un diseño de tratamientos factorial $2 \times 5$, los factores fueron los dos cultivares y cinco niveles de salinidad inducidos con cloruro de sodio $(\mathrm{NaCl})$. Los niveles de salinidad de la solución nutritiva se ajustaron a las conductividades eléctricas de 2.0, 2.5, 3.0 y 3.5 dS $\mathrm{m}^{-1}$ con adición de $\mathrm{NaCl}$; el testigo consistió en la solución nutritiva Steiner al $50 \% \operatorname{sin~} \mathrm{NaCl}$, que presentó CE de $1.2 \mathrm{dS}$ $\mathrm{m}^{-1}$. La cantidad de $\mathrm{NaCl}$ se calculó mediante la ecuación propuesta por el Laboratorio de Salinidad de los Estados Unidos de América (Richards, 1980): $\mathrm{mg} \mathrm{L}^{-1}=640 \times \mathrm{CE}$, donde CE es la conductividad eléctrica expresada en dS $\mathrm{m}^{-1}$. El diseño experimental utilizado fue completamente al azar con cinco repeticiones y la unidad experimental consistió de dos plantas.

\section{Variables evaluadas}

Cada 15 d se registró el volumen de agua transpirada, se realizaron dos evaluaciones del número y longitud de brotes en $\mathrm{cm}$ y número de hojas por brote a los 15 y 30 d después de la aplicación de los tratamientos. Después de cuatro meses, las plantas se trasladaron al laboratorio donde se procedió a separar la raíz, tallo y hojas para evaluar el resto de variables. Se cuantificó el volumen de agua transpirada (L/planta/semana), el número y longitud de brotes, número de hojas por brote, vida media de la hoja (en tres hojas por planta) colocando un anillo de estambre en el peciolo, se identificó la fecha cuando la hoja había abierto por el color del estambre y se contaron los días a abscisión; el contenido de clorofila se evaluó con un medidor de clorofila SPAD-502 (Konica Minolta, Tokio, Japón) en dos hojas maduras en la parte media de la planta, a los 99, 106 y 113 días de establecido el experimento (ddee).

Al final del experimento se medió el área foliar de cinco hojas en $\mathrm{cm}^{2}$ con un integrador de área foliar LI-3100 (LICOR, Inc. Lincoln, Nebraska, EUA). Para la impresión de estomas, de cada planta se tomaron tres hojas completas, maduras, sin daño mecánico, ni por insectos; la impresión de estomas se realizó en la parte media, abaxial de la hoja con el método microrelieve con pegamento de secado rápido (Weyers y Meidner, 1990), se fotografiaron tres campos por hoja con el objetivo 40x de un microscopio estereoscópico Leica CME (Microsystems AG, Wetzlar, Alemania); las imágenes se analizaron con el programa Image Tool V3.0 (UTHSCSA, University of Texas Health Science Center, San Antonio, Texas); se cuantificó el número de estomas por campo, la densidad estomática y se midió el diámetro longitudinal de cinco estomas elegidos al azar. A los 120 d se midió la longitud de raíz en $\mathrm{cm}$. Se determinó la materia fresca y seca en g en hojas, tallo y raíz, la concentración de P, Ca, K y Na en porcentaje, y se calculó la relación $\mathrm{K} / \mathrm{Na}$.

\section{Análisis estadístico}

Se realizó análisis de varianza y prueba de medias por Tukey $(P \leq 0.05)$. Los datos se procesaron con el programa SAS ${ }^{\circledR} 9.2$ (Statistical Analysis System, SAS Institute Inc.).

\section{RESULTADOS Y DISCUSIÓN}

Los modelos factoriales permiten analizar por separado el efecto de los factores principales y de la interacción sobre las variables respuesta. En este caso sólo se presentó interacción significativa en la acumulación de materia seca, por lo que únicamente se analizó el efecto de los factores principales de manera independiente, excepto en la variable mencionada. El análisis de varianza para las variables de crecimiento se muestra en el Cuadro 1.

A los 15 ddee, sólo se presentaron diferencias entre cultivares para longitud de brote. Merita presentó mayor longitud que Calvillo Siglo XXI (10.46 y 9.06 cm, respectivamente). Se ha demostrado que el genotipo influye no sólo en el tamaño de brote sino en la precocidad del fruto y desarrollo de la planta (Castillo-Martínez et al., 2005). A los 30 ddee sólo se encontraron diferencias en el número de brotes debidas a la concentración de sal común en el medio (Cuadros 1 y 2). Casierra-Posada (2006) encontró, en plantas de guayabo Palmira ICA-1 de 90 d, disminución de $30 \%$ del área foliar y $16.6 \%$ de peso seco a partir de $3.8 \mathrm{dS} \mathrm{m}^{-1}$ después de cuatro semanas bajo los tratamientos de salinidad.

La salinidad no afectó el tamaño y número de hojas por brote en las fechas evaluadas, a pesar de que produce sequía osmótica; esto significa que el crecimiento vegetativo se mantuvo de forma similar al testigo, lo que puede deberse a que las partes de las plantas que desarrollan los potenciales hídricos más bajos obtienen agua de los tejidos viejos, ya que diferentes partes de las plantas pierden agua a diferente velocidad y nivel (Pallardy, 2008).

\section{Consumo de agua}

El consumo de agua presentó diferencias estadísticas debido a las condiciones de salinidad que se manifestaron a partir de los $45 \mathrm{~d}$ (Cuadro 3).

Las plantas que consumieron más agua fueron las que se cultivaron en CE de $2.5 \mathrm{dS} \mathrm{m}^{-1}$, y presentaron también el mayor número de brotes (Cuadro 3). En ocasiones las raíces pueden absorber la sal del suelo para aumentar 
Cuadro 1. Media general, coeficiente de variación y significancia estadística para las variables evaluadas en genotipos de guayabo cultivados en diferentes condiciones de salinidad, 15 y 30 días después de establecido el experimento.

\begin{tabular}{|c|c|c|c|c|c|}
\hline \multirow{2}{*}{ Variable } & \multirow{2}{*}{ Media } & \multirow{2}{*}{ CV (\%) } & \multicolumn{3}{|c|}{$\operatorname{Pr}>\mathrm{F}$} \\
\hline & & & Cultivar & Salinidad & Cultivar $\times$ Salinidad \\
\hline \multicolumn{6}{|l|}{15 ddee } \\
\hline Número de brotes & 5.45 & 25.22 & 0.4709 & 0.6279 & 0.5962 \\
\hline Longitud de brote (cm) & 9.76 & 18.30 & $0.0457 *$ & 0.2563 & $0.0340 *$ \\
\hline Número de hojas/brote & 5.19 & 9.55 & 0.0620 & 0.1052 & $0.0306 *$ \\
\hline \multicolumn{6}{|l|}{30 ddee } \\
\hline Número de brotes & 5.79 & 23.34 & 0.9734 & $0.0238 *$ & 0.5958 \\
\hline Longitud de brote (cm) & 6.44 & 14.03 & 0.7604 & 0.9296 & $0.0458 *$ \\
\hline Número de hojas/brote & 2.82 & 19.56 & 0.6478 & 0.5804 & 0.0857 \\
\hline
\end{tabular}

Dde: días después de establecido el experimento, $\mathrm{CV}$ : coeficiente de variación. *: significativo $(\mathrm{P} \leq 0.05)$.

Cuadro 2. Número promedio de brotes por planta y vida media de la hoja en dos cultivares de guayabo sometido a diferentes condiciones de salinidad.

\begin{tabular}{lcc}
\hline $\begin{array}{l}\text { Salinidad } \\
\left(\mathrm{dS} \mathrm{m}^{-1}\right)\end{array}$ & $\begin{array}{c}\text { Número de brotes } \\
\text { por planta 30 ddee }\end{array}$ & $\begin{array}{c}\text { Vida media de la } \\
\text { hoja }(\mathrm{d})\end{array}$ \\
\hline 1.2 & $5.58 \mathrm{ab}$ & $74.17 \mathrm{a}$ \\
2.0 & $6.29 \mathrm{ab}$ & $71.67 \mathrm{a}$ \\
2.5 & $7.08 \mathrm{a}$ & $62.17 \mathrm{~b}$ \\
3.0 & $5.75 \mathrm{ab}$ & $52.83 \mathrm{c}$ \\
3.5 & $4.25 \mathrm{~b}$ & $42.50 \mathrm{~d}$ \\
CV $(\%)$ & 23.34 & 6.97 \\
DSH (0.05) & 2.03 & 7.30 \\
\hline
\end{tabular}

Medias con letras iguales en la misma columna no difieren estadísticamente (Tukey, $\mathrm{P} \leq 0.05$ ). CV: coeficiente de variación, DSH: diferencia significativa honesta.

la presión osmótica, lo que incrementa la capacidad de absorber agua (Zhuang et al., 2010). La disminución en la transpiración en las plantas cultivadas en concentraciones de más alta salinidad pudo deberse a que las plantas reducen la transpiración para evitar la absorción excesiva de iones tóxicos y su acumulación en los tejidos. De la misma forma, Casierra-Posada (2006) encontró en plantas de guayabo cv. Cimpa 00916 de tres meses de edad propagadas por semilla un ligero incremento en la evapotranspiración (0.03\%) a $2.0 \mathrm{mM}$ de $\mathrm{NaCl}$; sin embargo, en concentraciones de 4.0,6.0 y $8.0 \mathrm{mM}$ la evapotranspiración se redujo.

\section{Contenido de clorofila}

Sólo se encontraron diferencias en el contenido de clorofila entre cultivares a los 113 ddee. Esto significa que, para esta variable, el comportamiento de los cultivares difiere en el tiempo (Cuadro 4). Al contrario de lo consignado por Gonzalez et al. (2012), quienes encontraron que plantas de tres meses de portainjertos de cítricos, limón Rugoso y citrumelo Swingle, a las que se les agregaron $50 \mathrm{mM}$ de $\mathrm{NaCl}$ en la solución nutritiva, disminuyeron el índice de clorofila a los 40 ddee de 49 a 23 y de 62 a 50 unidades SPAD. Es importante considerar que la respuesta al estrés depende del genotipo y del tiempo que permanecen las plantas en esas condiciones (Tester y Davenport, 2003), lo que podría explicar la disminución del contenido de clorofila a través del tiempo en hojas del cv. Merita observada en este trabajo.

\section{Vida media de la hoja}

La vida media de la hoja (Cuadro 2) disminuyó conforme se incrementó la salinidad. Estos resultados coinciden con lo reportado por Plaut et al. (2000), quienes encontraron que la salinidad incrementó el área foliar necrosada y la senescencia de la hoja en Saccharum officinarum L.

El número de días a caída de hojas en los diferentes tratamientos fue ligeramente menor de la mitad de lo reportado por Kumar y Pandey (1979); estos autores encontraron en guayabo cv. Lucknow-49 que la estabilización de nutrimentos ocurrió entre los cinco y siete meses después de emergida la hoja. 
Cuadro 3. Consumo promedio de agua (L/planta/semana) en plantas de dos cultivares de guayabo sometidas a diferentes condiciones de salinidad.

\begin{tabular}{lcccccccc}
\hline \multirow{2}{*}{ Salinidad $\left(\mathrm{dS} \mathrm{m}{ }^{-1}\right)$} & \multicolumn{7}{c}{ Días después de establecido el experimento } \\
\cline { 2 - 9 } & 15 & 30 & 45 & 60 & 75 & 90 & 105 & 120 \\
\hline 1.2 & $0.82 \mathrm{a}$ & $0.85 \mathrm{a}$ & $0.69 \mathrm{ab}$ & $0.92 \mathrm{~b}$ & $0.94 \mathrm{~b}$ & $1.67 \mathrm{a}$ & $0.92 \mathrm{~b}$ & $0.92 \mathrm{~b}$ \\
2.0 & $0.75 \mathrm{a}$ & $0.75 \mathrm{a}$ & $0.70 \mathrm{ab}$ & $0.75 \mathrm{~b}$ & $0.75 \mathrm{~b}$ & $0.75 \mathrm{~b}$ & $0.75 \mathrm{~b}$ & $0.75 \mathrm{~b}$ \\
2.5 & $0.78 \mathrm{a}$ & $0.58 \mathrm{a}$ & $0.81 \mathrm{a}$ & $1.58 \mathrm{a}$ & $1.58 \mathrm{a}$ & $1.58 \mathrm{a}$ & $1.58 \mathrm{a}$ & $1.60 \mathrm{a}$ \\
3.0 & $0.83 \mathrm{a}$ & $0.83 \mathrm{a}$ & $0.72 \mathrm{ab}$ & $0.83 \mathrm{~b}$ & $0.83 \mathrm{~b}$ & $0.81 \mathrm{~b}$ & $0.85 \mathrm{~b}$ & $0.85 \mathrm{~b}$ \\
3.5 & $0.56 \mathrm{a}$ & $0.56 \mathrm{a}$ & $0.55 \mathrm{~b}$ & $0.56 \mathrm{~b}$ & $0.58 \mathrm{~b}$ & $0.58 \mathrm{~b}$ & $0.58 \mathrm{~b}$ & $0.51 \mathrm{~b}$ \\
CV (\%) & 38.45 & 37.81 & 35.54 & 35.27 & 35.27 & 39.52 & 35.27 & 36.54 \\
DSH (0.05) & 6.13 & 3.02 & 0.56 & 0.57 & 0.57 & 0.74 & 0.57 & 0.57 \\
\hline
\end{tabular}

Medias con letras iguales en la misma columna no difieren estadísticamente (Tukey, P $\leq$ 0.05). CV: coeficiente de variación, DSH: diferencia significativa honesta.

Cuadro 4. Contenido de clorofila a los 99, 106 y 113 días de establecido el experimento y materia seca en hoja y raíz en dos cultivares de guayabo.

\begin{tabular}{|c|c|c|c|c|c|}
\hline \multirow{3}{*}{ Cultivar } & \multicolumn{3}{|c|}{ Contenido de clorofila (unidades SPAD) } & \multicolumn{2}{|c|}{ Materia seca (g) } \\
\hline & \multicolumn{3}{|c|}{ ddee } & \multicolumn{2}{|c|}{ Estructura } \\
\hline & 99 & 106 & 113 & Hoja & Raíz \\
\hline Calvillo Siglo XXI & $42.29 a$ & $39.23 \mathrm{a}$ & $36.35 a$ & $59.21 \mathrm{a}$ & $35.76 a$ \\
\hline Merita & $42.04 \mathrm{a}$ & 39.09 a & $32.74 b$ & $51.54 \mathrm{a}$ & $17.96 b$ \\
\hline CV $(\%)$ & 3.98 & 6.54 & 6.98 & 25.88 & 13.69 \\
\hline DSH (0.05) & 3.59 & 3.20 & 2.65 & 13.98 & 6.46 \\
\hline
\end{tabular}

Medias con letras iguales en la misma columna no difieren estadísticamente (Tukey, $\mathrm{P} \leq 0.05)$. dde: días después de establecido el experimento, CV: coeficiente de variación, DSH: diferencia significativa honesta.

\section{Número de hojas y área foliar}

No se encontraron diferencias en el área foliar de cinco hojas (Cuadro 5). Aunque se considera que el área foliar puede ser afectada por la salinidad, la respuesta depende de los cultivares y su grado de tolerancia (Ali et al., 2004).

\section{Número de hojas, longitud de raíz y materia fresca}

No se encontraron diferencias en el número de hojas, longitud de raíz, ni materia fresca (Cuadro 5). Se considera que la salinidad provoca decremento en el crecimiento de las plantas debido a la disminución de la absorción de agua, y el contenido de materia fresca es más afectado que el de materia seca; ésto resulta en menor tamaño y, en ocasiones, menor número de hojas (Al-Maskri et al., 2010). En este trabajo, el número de hojas no fue afectado, lo que demuestra que los cultivares presentaron cierta tolerancia a las condiciones salinas en los niveles evaluados.

\section{Materia seca}

El análisis de varianza no mostró diferencias en materia seca de hoja y raíz (Cuadro 5) pero sí interacción genotipo × salinidad en materia seca del tallo. En la Figura 1 se puede observar que la respuesta a las condiciones de salinidad fue diferente entre cultivares, lo que puede ser explicado por las diferencias en las características genotípicas que, por mínimas que sean, pueden causar diferencias relacionadas con el aumento de la tolerancia al estrés salino (Yeo, 1998).

\section{Concentración de nutrimentos en hoja y raíz}

El cv. Merita presentó mayor concentración de P y Ca en hojas que Calvillo Siglo XXI (Cuadro 6); ésto difiere de lo encontrado por Guerra y Bautista (2002), quienes reportaron la misma concentración foliar de N, P, K, Ca, $\mathrm{Mg}, \mathrm{Zn}, \mathrm{Fe}, \mathrm{Cu}$ y $\mathrm{Mn}$ en tres clones de guayabo (Mara 4, Mara 6 y Mara 8); ellos reportaron concentraciones de 
Cuadro 5. Media general, coeficiente de variación y significancia estadística de las variables evaluadas en dos cultivares de guayabo sometidos a diferentes condiciones de salinidad.

\begin{tabular}{|c|c|c|c|c|c|}
\hline \multirow{2}{*}{ Variable } & \multirow{2}{*}{ Media } & \multirow{2}{*}{ CV (\%) } & \multicolumn{3}{|c|}{$\operatorname{Pr}>\mathrm{F}$} \\
\hline & & & Cultivar & Salinidad & Cultivar $\times$ Salinidad \\
\hline Área foliar (cm²) & 244.63 & 14.87 & 0.5952 & 0.1922 & 0.7860 \\
\hline Número de hojas & 114.51 & 30.37 & 0.4509 & 0.3330 & 0.5304 \\
\hline Longitud de raíz (cm) & 34.78 & 33.89 & 0.1403 & 0.9955 & 0.8600 \\
\hline Materia fresca hoja (g) & 164.06 & 39.09 & 0.3143 & 0.1922 & 0.5792 \\
\hline Materia fresca tallo (g) & 200.15 & 33.23 & 0.2746 & 0.4578 & 0.4456 \\
\hline Materia fresca raíz (g) & 92.34 & 42.06 & 0.7843 & 0.8747 & 0.2382 \\
\hline Materia seca hoja (g) & 45.43 & 40.38 & 0.4667 & 0.1020 & 0.2727 \\
\hline Materia seca tallo (g) & 100.75 & 31.58 & $0.0315 *$ & $0.0210 *$ & $0.0332 *$ \\
\hline Materia seca raíz (g) & 21.55 & 39.35 & 0.3192 & 0.3995 & 0.8583 \\
\hline
\end{tabular}

CV: coeficiente de variación, $*$ : significancia estadística $(P \leq 0.05)$.

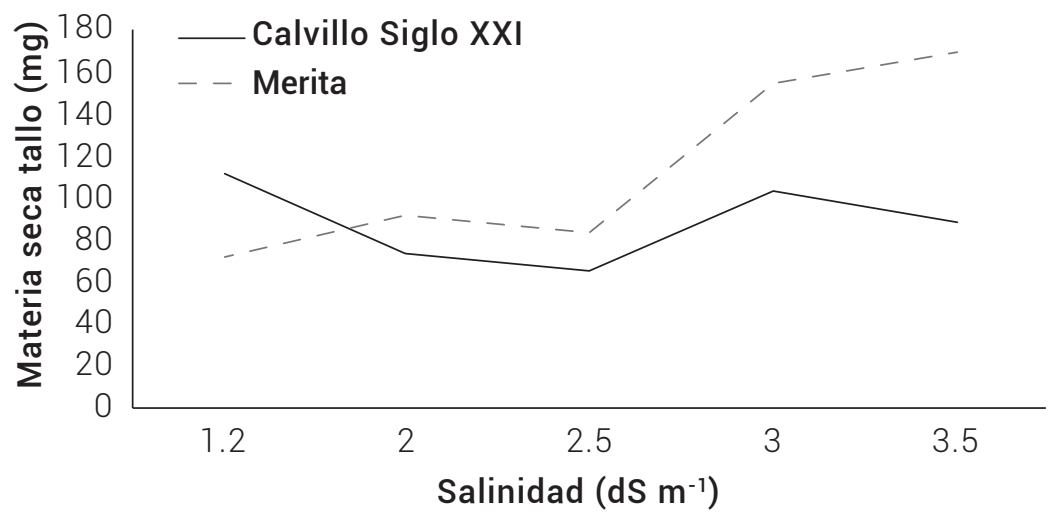

Figura 1. Interacción entre los factores cultivar y conductividad eléctrica en la acumulación de materia seca en el tallo.

$0.18 \%$ de $\mathrm{P}, 1.27$ a $1.25 \%$ de $\mathrm{K}$ y 1.62 a $1.67 \%$ de Ca. Estas concentraciones difieren de lo encontrado en esta investigación, ya que en los dos materiales probados la concentración de P fue 28 y $38 \%$ menor, el de K casi el doble y de Ca presentaron 40 y $55 \%$ menos de lo reportado por estos autores.

El resultado de la prueba de medias para el porcentaje de los nutrimentos evaluados en hojas y raíz en las diferentes condiciones de salinidad se muestra en el Cuadro 7.

La concentración de $\mathrm{Na}^{+}$en hoja se incrementó con la salinidad (Cuadro 7). Las plantas transportan $\mathrm{Na}^{+}$a las hojas como un mecanismo de eliminación de sales (Rajput et al., 2015) y la toxicidad por $\mathrm{Na}^{+}$pudo ser la causa de que se acelerara la senescencia de las hojas. El transporte de $\mathrm{Na}^{+}$hacia las hojas queda en evidencia, ya que no se presentó incremento significativo de $\mathrm{Na}^{+}$en la raíz en las diferentes condiciones de salinidad. Aunque las plantas presentan selectividad alta de $\mathrm{K}^{+}$en relación con $\mathrm{Na}^{+}$(Goldhamer y Viveros, 2000), en condiciones salinas el $\mathrm{Na}^{+}$compite con el $\mathrm{K}^{+}$en los cotransportadores $\mathrm{Na}^{+}-\mathrm{K}^{+}$ (Zhu, 2003). El transporte de $\mathrm{Na}^{+}$a las hojas observado en este estudio pudo evitar la competencia por los cotransportadores $\mathrm{Na}^{+}-\mathrm{K}^{+}$.

Conforme se incrementó la concentración de $\mathrm{Na}^{+}$ disminuyó la concentración de $\mathrm{K}^{+}$en la hoja (Cuadro 7); sin embargo, a $2.5 \mathrm{dS} \mathrm{m}^{-1}$ la concentración de $\mathrm{K}^{+}$en la hoja disminuyó en únicamente 6.98 \% y fue estadísticamente igual al testigo (CE de $1.2 \mathrm{dS} \mathrm{m}^{-1}$ ); mientras que en raíz, con esa CE, la reducción fue de 41.67 \% y llegó a 55.55 $\%$ a los $3.5 \mathrm{dS} \mathrm{m}^{-1}$ en comparación con el testigo; ésto significa que, junto con el $\mathrm{Na}^{+}$, la planta transportó el $\mathrm{K}^{+}$ hacia las hojas y disminuyó la toxicidad metabólica que ocurre cuando el $\mathrm{Na}^{+}$compite con el $\mathrm{K}^{+}$por los sitios de 
Cuadro 6. Concentración promedio de $\mathrm{P}, \mathrm{K}, \mathrm{Ca}$, Na y relación $\mathrm{K} / \mathrm{Na}$ en hojas de dos cultivares de guayabo.

\begin{tabular}{lccccc}
\hline Cultivar & $\mathrm{P}(\%)$ & $\mathrm{K}(\%)$ & $\mathrm{Ca}(\%)$ & $\mathrm{Na}(\%)$ & Relación K/Na \\
\hline Merita & $0.13 \mathrm{a}$ & $2.49 \mathrm{a}$ & $1.05 \mathrm{a}$ & $6.89 \mathrm{a}$ & $1.08 \mathrm{a}$ \\
C. Siglo XXI & $0.11 \mathrm{~b}$ & $2.42 \mathrm{a}$ & $0.75 \mathrm{~b}$ & $6.35 \mathrm{a}$ & $1.05 \mathrm{a}$ \\
CV (\%) & 17.39 & 18.67 & 43.17 & 40.17 & 40.99 \\
DSH (0.05) & 0.15 & 0.35 & 0.29 & 2.03 & 0.61
\end{tabular}

Medias con letras iguales en la misma columna no difieren estadísticamente (Tukey, $\mathrm{P} \leq 0.05)$. CV: coeficiente de variación, DSH: diferencia significativa honesta.

Cuadro 7. Concentración promedio de $\mathrm{K}, \mathrm{Na}$ y relación $\mathrm{K} / \mathrm{Na}$ en hojas y raíz en dos cultivares de guayabo sometidos a diferentes condiciones de salinidad.

\begin{tabular}{|c|c|c|c|c|c|c|}
\hline \multirow{2}{*}{$\begin{array}{c}\text { Salinidad } \\
\left(\mathrm{dS} \mathrm{m}^{-1}\right)\end{array}$} & \multicolumn{2}{|c|}{ Hojas } & \multicolumn{4}{|c|}{ Raíz } \\
\hline & $\mathrm{K}(\%)$ & $\mathrm{Na}(\%)$ & $\mathrm{K} / \mathrm{Na}$ & $K(\%)$ & $\mathrm{Na}(\%)$ & $\mathrm{K} / \mathrm{Na}$ \\
\hline 1.2 & $2.85 \mathrm{a}$ & $3.19 \mathrm{c}$ & $1.78 \mathrm{a}$ & $1.37 \mathrm{a}$ & $0.88 \mathrm{a}$ & $1.35 \mathrm{a}$ \\
\hline 2.0 & $2.60 a b$ & 5.22 bc & $1.28 a b$ & $1.14 \mathrm{ab}$ & $1.07 \mathrm{a}$ & $0.52 b$ \\
\hline 2.5 & $2.65 a b$ & $6.09 a b c$ & $1.19 a b$ & $0.80 \mathrm{bc}$ & $1.23 \mathrm{a}$ & $0.46 b$ \\
\hline 3.0 & $2.36 a b$ & $8.42 a b$ & $0.69 a b$ & $0.91 \mathrm{abc}$ & $1.37 \mathrm{a}$ & $0.31 \mathrm{~b}$ \\
\hline 3.5 & $1.94 \mathrm{~b}$ & $10.16 \mathrm{a}$ & $0.39 \mathrm{~b}$ & $0.61 \mathrm{c}$ & $1.72 \mathrm{a}$ & $0.22 b$ \\
\hline CV (\%) & 18.67 & 40.17 & 40.99 & 28.28 & 40.09 & 40.44 \\
\hline DSH (0.05) & 0.80 & 2.03 & 1.38 & 0.36 & 0.93 & 0.71 \\
\hline
\end{tabular}

Medias con letras iguales en la misma columna no difieren estadísticamente (Tukey, $\mathrm{P} \leq 0.05)$. CV: coeficiente de variación, DSH: diferencia significativa honesta.

unión (Tester y Davenport, 2003). González-Jiménez et al. (2020) también reportaron acumulación de K y S en la hoja de fresa cultivada en condiciones salinas, aunque la concentración de Na no se vio afectada por la salinidad.

En el caso de plántulas de guayabo cv. Safeda en condiciones salinas, Singh et al. (2015) encontraron incremento de aproximadamente $30 \%$ en la concentración de $\mathrm{K}$ en hojas cuando el nivel de salinidad fue de $1.4 \mathrm{dS}$ $\mathrm{m}^{-1}$ en comparación con CE menores y mayores (0.5, 0.98 y $2 \mathrm{dS}^{-1}$ ). Lo anterior se atribuye a la absorción diferencial de nutrimentos, lo que permite a la planta mantener sus procesos metabólicos. En este caso, la concentración de $\mathrm{K}^{+}$que presentan las hojas es alta en comparación con lo reportado en otros cultivares, lo que puede ser la razón de que las plantas mantuvieran las condiciones necesarias para los procesos metabólicos y no manifestaran diferencias en la mayoría de las variables evaluadas, incluyendo los nutrimentos, ya que la salinidad no afectó la concentración de P y Ca en hojas o raíces. La disponibilidad de $\mathrm{K}$ para contrarrestar el efecto de la salinidad es importante y se ha propuesto que, para atenuar el efecto adverso de las sales en el rendimiento de los cultivos, se utilice la fertilización potásica, la cual permite regular la competencia por los nutrimentos que provoca el $\mathrm{Na}^{+}$(Munns, 2005).
En cuanto a la relación $\mathrm{K} / \mathrm{Na}$, se encontraron diferencias debidas a la salinidad. Se observa en el Cuadro 7 que en la hoja la relación $\mathrm{K} / \mathrm{Na}$ disminuyó progresivamente con


inferior al resto de tratamientos, mientras que en la raíz

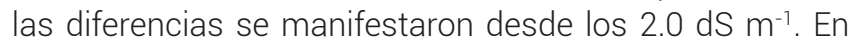
este trabajo la relación media $\mathrm{K} / \mathrm{Na}$ en hojas para todas las CE fue de 1.065, mientras que en raíz fue de 0.573 . Se considera que los genotipos tolerantes a salinidad generalmente muestran una mayor relación $\mathrm{K} / \mathrm{Na}$ en los brotes y menor en las raíces (Bağci et al., 2007).

Mantener una adecuada relación K/Na es de suma importancia en la planta cuando ésta se encuentra bajo estrés por sales. Aunque Bağci et al. (2003) indican que se debe dar más importancia a una alta absorción de $\mathrm{K}$ y después a la relación $\mathrm{K} / \mathrm{Na}$, esta relación puede ser afectada por diferencias no sólo en la absorción de $\mathrm{K}^{+} \mathrm{y}$ $\mathrm{Na}^{+}$, sino en la distribución de estos elementos en brotes y raíces. En este caso, la movilización del $\mathrm{Na}^{+}$hacia las hojas mantuvo una relación $\mathrm{K} / \mathrm{Na}$ en la raíz que permitió que las plantas mostraran mayor tolerancia a las condiciones de salinidad. 


\section{Densidad y longitud de estomas}

No se encontraron diferencias en el número y longitud estomas entre cultivares ni entre condiciones de salinidad. La ausencia de diferencias significativas pudiera explicarse por la tolerancia a sales debida a la presencia de tricomas en las hojas en los cultivares evaluados. Aunque en este trabajo no se consideró como variable a la densidad de tricomas, la cantidad de tricomas presente en las hojas dificultó la toma de impresiones. La presencia de tricomas en las hojas se ha relacionado con la tolerancia a salinidad, debido a que éstas disminuyen la difusión de gases (Quintana et al., 2012).

Por último, la resistencia a salinidad se ha relacionado con la madurez del tejido, y aunque se reportó que la salinidad afectó la germinación de semillas de guayabo (Casierra-Posada, 2006) y el desarrollo temprano de las plántulas (Sá et al., 2016), las plantas evaluadas obtenidas a partir de estacas maduras presentaron mayor tolerancia a las CE evaluadas por un periodo de cuatro meses.

\section{CONCLUSIONES}

La salinidad no tuvo efecto significativo en el crecimiento de las plantas de guayabo obtenidas a partir de material maduro en los cuatro meses que duró el experimento; sin embargo, sí aceleró la senescencia de la hoja. La capacidad de los dos cultivares de mantener alta la concentración de $\mathrm{K}^{+}$en la hoja, así como el transporte del $\mathrm{Na}^{+}$hacia las hojas, incrementó la resistencia a la salinidad en los niveles evaluados, al mantener un adecuado suministro de agua por la raíz. El cultivar Calvillo Siglo XXI presentó mayor contenido de clorofila a los 113 días después de establecido el experimento, mientras que Merita presentó mayor concentración de P y Ca en la hoja.

\section{AGRADECIMIENTOS}

Los autores agradecen al M.C. Roberto Sánchez Lucio, al INIFAP y a la Red de Guayabo de SINAREFI por el material biológico proporcionado para la presente investigación.

\section{BIBLIOGRAFÍA}

Al-Maskri A., L. Al-Kharusi, H. Al-Miqbali and M. M. Khan (2010) Effects of salinity stress on growth of lettuce (Lactuca sativa) under closed-recycle nutrient film technique. International Journal of Agriculture and Biology 12:377-380.

Ali Y., Z. Aslam, M. Y. Ashraf and G. R. Tahir (2004) Effect of salinity on chlorophyll concentration, leaf area, yield and yield components of rice genotypes grown under saline environment. International Journal of Environmental Science and Technology 1:221-225, https://doi.org/10.1007/BF03325836

Bağci S. A., H. Ekiz and A. Yilmaz (2003) Determination of the salt tolerance of some barley genotypes and the characteristics affecting tolerance. Turkish Journal of Agriculture and Forestry
27:253-260.

Bağci S. A., H. Ekiz and A. Yilmaz (2007) Salt tolerance of sixteen wheat genotypes during seedling growth. Turkish Journal of Agriculture and Forestry 31:363-372.

Casierra-Posada F. (2006) Distribución y producción total de materia seca en guayabo (Psidium guajava L. Cv. Palmira ICA-1) bajo estrés salino. Revista Orinoquia 10:59-66.

Castillo-Martínez R., M. Livera-Muñoz y G. J. Márquez-Guzmán (2005) Caracterización morfológica y compatibilidad sexual de cinco genotipos de pitahaya (Hylocereus undatus). Agrociencia 39:183-194.

Cha-Um S. and C. Kirdmanee (2009) Effect of salt stress on proline accumulation, photosynthetic ability and growth characters in two maize cultivars. Pakistan Journal of Botany 41:87-98.

Goldhamer D. A. and M. Viveros (2000) Effects of preharvest irrigation cutoff durations and postharvest water deprivation on almond tree performance. Irrigation Science 19:125-131, https://doi. org/10.1007/s002710000013

Gonzalez P., J. P. Syvertsen and E. Etxeberria (2012) Sodium distribution in salt-stressed Citrus rootstock seedlings. HortScience 47:15041511, https://doi.org/10.21273/HORTSCI.47.10.1504

González-Jiménez S. L., A. M. Castillo-González, M. R. García-Mateos, L. A. Valdez-Aguilar, C. Ybarra-Moncada y E. Avitia-García (2020) Respuesta de fresa cv. Festival a la salinidad. Revista Fitotecnia Mexicana 43:53-60, https://doi.org/10.35196/rfm.2020.1.5340

Guerra E. y D. Bautista (2002) Contenido foliar de elementos nutricionales en tres clones de guayaba (Psidium guajava L.) en época de alta actividad de crecimiento. Bioagro 14:99-104.

Kumar P. and R. M. Pandey (1979) Sampling for mineral content in leaves of guava cultivar Lucknow 49. Scientia Horticulturae 11:163174, https://doi.org/10.1016/0304-4238(79)90041-4

Marschner H. (2002) Mineral Nutrition of Higher Plants. Second edition. Academic Press. London. 889 p.

Munns R. (2005) Genes and salt tolerance: bringing them together. New Phytologist 167:645-663, https://doi.org/10.1111/j.14698137.2005.01487.x

Munns R. and M. Tester (2008) Mechanisms of salinity tolerance. Annual Review of Plant Biology 59:651-681, https://doi.org/10.1146/ annurev.arplant.59.032607.092911

Nawaz K., K. Hussain, A. Majeed, F. Khan, S. Afghan and K. Ali (2010) Fatality of salt stress to plants: morphological, physiological and biochemical aspects. African Journal of Biotechnology 9:54755480 .

Pallardy S. G. (2008) Physiology of Woody Plants. Third edition Academic Press. San Diego, California, USA. 454 p.

Plaut Z., F. C. Meinzer and E. Federman (2000) Leaf development, transpiration and ion uptake and distribution in sugarcane cultivars grown under salinity. Plant and Soil 218:59-69, https:// doi.org/10.1023/A:1014996229436

Quintana C. G., M. E. Sanabria, M. G. Ojeda y R. E. Valera (2012) Efecto de la salinidad sobre la anatomía foliar y radical de la piña (Ananas comosus (L.) Merr.) 'Española roja'. Pittieria 36:87-108.

Rajput V. D., Y. Chen and M. Ayup (2015) Effects of high salinity on physiological and anatomical indices in the early stages of Populus euphratica growth. Russian Journal of Plant Physiology 62:229-236, https://doi.org/10.1134/S1021443715020168

Richards L. A. (1980) Diagnóstico y Rehabilitación de Suelos Salinos y Sódicos. Sexta edición. Limusa. México, D. F. 172 p.

Ruiz E. F. H., R. L. Villalpando G., B. Murillo A., F. A. Beltrán M. y L. G. Hernández M. (2014) Respuesta diferencial a la salinidad de genotipos de tomate (Lycopersicon esculentum Mill.) en primeras etapas fenológicas. Terra Latinoamericana 32:311-323.

Sá F. V. S., R. G. Nobre, L. A. Silva, R. C. L. Moreira, E. P. de Paiva and F. A. de Oliveira (2016) Tolerance of guava rootstocks under salt stress. Revista Brasileira de Engenharia Agrícola e Ambiental 20:1072-1077, https://doi.org/10.1590/1807-1929/agriambi. v20n12p1072-1077

SIAP, Servicio de Información Agroalimentaria y Pesquera (2018) Atlas agroalimentario 2012-2018. Servicio de Información Agroalimentaria y Pesquera, Secretaría de Agricultura, Desarrollo Rural, Pesca y Alimentación. Ciudad de México. 222 p. https://nube.siap.gob.mx/gobmx_publicaciones_siap/ 
pag/2018/Atlas-Agroalimentario-2018 (Noviembre 2018)

Singh M., J. Kumar, S. Singh, V. P. Singh and S. M. Prasad (2015) Roles of osmoprotectants in improving salinity and drought tolerance in plants: a review. Reviews in Environmental Science and Bio/ Technology 14:407-426, https://doi.org/10.1007/s11157-0159372-8

Singh A., A. Kumar, R. K. Yadav, A. Dutta and D. K. Sharma (2016) Growth and mineral nutrition in salt stressed guava (Psidium guajava L.) cv. Allahabad Safeda. Journal of AgriSearch 3:21-25, https:// doi.org/10.21921/jas.v3i1.11403

Steiner A. A. (1984) The universal nutrient solution. In: Proceedings of the Sixth International Congress on Soilless Culture. International Society for Soilless Culture. Wageningen, The Netherlands. pp:633-650

Tester M. and R. Davenport (2003) $\mathrm{Na}^{+}$tolerance and $\mathrm{Na}^{+}$transport in higher plants. Annals of Botany 91:503-527, https://doi org/10.1093/aob/mcg058

Weyers J. D. B. and H. Meidner (1990) Methods in Stomatal Research. Longman Scientific \& Technical. New York. 233 p.

Yeo A. (1998) Predicting the interaction between the effects of salinity and climate change on crop plants. Scientia Horticuturae 78:159-174, https://doi.org/10.1016/S0304-4238(98)00193-9

Zhu J. K. (2003) Regulation of ion homeostasis under salt stress. Current Opinion in Plant Biology 6:441-445, https://doi.org/10.1016/ S1369-5266(03)00085-2

Zhuang L., W. H. Li, F. Yuan, W. C. Gong and Z. P. Tian (2010) Ecological adaptation characteristics of Populus euphratica and Tamarix ramosissima leaf microstructures in the lower reaches of Tarim River. Acta Ecologica Sinica 30:62-66, https://doi.org/10.1016/j. chnaes.2010.03.003 
